\title{
Posttraumatic stress due to an acute coronary syndrome increases risk of 42-month major adverse cardiac events and all- cause mortality
}

Donald Edmondson, Ph.D. ${ }^{1}$, Nina Rieckmann, Ph.D. ${ }^{2}$, Jonathan A. Shaffer, Ph.D. ${ }^{1}$, Joseph E. Schwartz, Ph.D. ${ }^{1}$, Matthew M. Burg, Ph.D. ${ }^{1,3}$, Karina W. Davidson, Ph.D. ${ }^{1}$, Lynn Clemow, Ph.D. ${ }^{1}$, Daichi Shimbo, M.D. ${ }^{1}$, and lan M. Kronish, M.D., M.P.H. ${ }^{4}$

${ }^{1}$ Center for Behavioral Cardiovascular Health, Columbia University Medical Center, New York

${ }^{2}$ Berlin School of Public Health, Charité University Medical Center, Berlin

${ }^{3}$ Section of Cardiovascular Medicine, Yale University School of Medicine, New Haven, CT

${ }^{4}$ Division of General Internal Medicine, Mount Sinai Medical Center, New York

\section{Abstract}

Approximately $15 \%$ of patients with acute coronary syndromes (ACS) develop posttraumatic stress disorder (PTSD) due to their ACS event. We assessed whether ACS-induced PTSD symptoms increase risk for major adverse cardiac events (MACE) and all-cause mortality (ACM) in an observational cohort study of 247 patients (aged 25-93 years; 45\% women) hospitalized for an ACS at one of 3 academic medical centers in New York and Connecticut between November 2003 and June 2005. Within 1 week of admission, patient demographics, Global Registry of Acute Coronary Events risk score, Charlson comorbidity index, left ventricular ejection fraction, and depression status were obtained. At 1-month follow-up, ACS-induced PTSD symptoms were assessed with the Impact of Events Scale-Revised. The primary endpoint was combined MACE (hospitalization for myocardial infarction, unstable angina or urgent/emergency coronary revascularization procedures) and ACM, which were actively surveyed for 42 months after index event. Thirty-six (15\%) patients had elevated intrusion symptoms, 32 (13\%) elevated avoidance symptoms, and $21(9 \%)$ elevated hyperarousal symptoms. Study physicians adjudicated 21 MACEs and 15 deaths during the follow-up period. In unadjusted Cox proportional hazards regression analyses, and analyses adjusted for sex, age, clinical characteristics and depression, high intrusion symptoms were associated with the primary endpoint (adjusted hazard ratio, 3.38; $95 \%$ confidence interval, $1.27-9.02 ; p=.015$ ). Avoidance and hyperarousal symptoms were not associated with the primary endpoint. The presence of intrusion symptoms is a strong and independent predictor of elevated risk for MACE and ACM, and should be considered in the risk stratification of ACS patients.

(C) 2011 Elsevier Ltd. All rights reserved.

Address for correspondence: Donald Edmondson, Ph.D., Center for Behavioral Cardiovascular Health, Columbia University Medical Center, 622 West 168 Street, PH9-948, New York, NY 10032; donald.edmondson@ columbia.edu; phone: 212-342-3674; fax: 212-305-3172.

Publisher's Disclaimer: This is a PDF file of an unedited manuscript that has been accepted for publication. As a service to our customers we are providing this early version of the manuscript. The manuscript will undergo copyediting, typesetting, and review of the resulting proof before it is published in its final citable form. Please note that during the production process errors may be discovered which could affect the content, and all legal disclaimers that apply to the journal pertain. 


\section{Keywords}

posttraumatic stress disorder; psychosocial factors; risk factors; acute coronary syndrome; recurrence; behavioral medicine

The experience of an acute coronary syndrome [ACS, i.e., myocardial infarction (MI), unstable angina (UA)] can be traumatic for many patients (Doerfler \& Paraskos, 2011). In fact, approximately $15 \%$ of patients develop significant symptoms of posttraumatic stress disorder (PTSD) due to an ACS (Ayers, Copland, \& Dunmore, 2009; Gander \& von Kanel, 2006). PTSD that occurs as a result of trauma in non-medical settings is associated with increased risk for incident cardiovascular disease (CVD) (Falger et al., 1992; Kubzansky \& Koenen, 2009). Furthermore, a growing body of literature suggests that PTSD due to an ACS can increase risk of ACS recurrence. The presence of PTSD due to an ACS has been associated with increased cardiovascular hospital readmission(Shemesh et al., 2004), lower treatment adherence (Shemesh et al., 2006; Shemesh et al., 2001), and poorer quality of life (Doerfler, Paraskos, \& Piniarski, 2005; Doerfler, Pbert, \& DeCosimo, 1994; Gamper et al., 2004; Ladwig et al., 1999). Indeed, a recent study of Swiss post-MI patients found a $42 \%$ increased risk of nonfatal ACS recurrence associated with each 10-point higher level of PTSD symptoms (von Kanel et al., 2011). However, previous research has either considered only nonfatal hospital readmissions, failed to control for potential confounders of the relationship between PTSD and outcomes including medical comorbidities and depression (an established psychosocial predictor of ACS recurrence), or both. Further, previous research has focused on only one category of ACS patients (e.g., MI only), rather than considering a sample of diverse ACS diagnoses.

The diagnosis of PTSD consists of symptoms that have been organized into three clusters: intrusions, avoidance, and hyperarousal (King, Leskin, King, \& Weathers, 1998). Intrusion symptoms include intrusive thoughts or images related to the traumatic event, nightmares, and flashbacks; avoidance symptoms include efforts to avoid stimuli related to the traumatic event; and hyperarousal symptoms include difficulty concentrating, insomnia, and hypervigilance to environmental threats. As with other psychiatric disorders known to influence cardiovascular outcomes, specific symptoms or features of the disorder may carry the bulk of the cardiovascular risk whereas others may not (Davidson et al., 2010). The independent relationships of PTSD symptom clusters to post-ACS prognosis has not been previously reported, though recent evidence suggests that intrusion symptoms may be the most important cluster for predicting CVD risk (Newton, Parker, \& Ho, 2005; von Kanel et al., 2011; von Kanel et al., 2008). It is particularly important to adjust for depression when assessing the independent relationships between PTSD symptom clusters and adverse outcomes, given that the two disorders are often comorbid, and individuals with depression often score similarly to those with PTSD on avoidance and especially hyperarousal (i.e., more so than intrusions, which appear to be relatively unique to PTSD) (Shalev et al., 1998).

The aim of this study was to explore the relationship of PTSD symptom clusters with recurrent cardiac events and all-cause mortality after ACS. Consistent with the literature discussed above, we hypothesized that the intrusions symptoms cluster would be most strongly associated with cardiovascular risk and all-cause mortality among patients who develop elevated PTSD symptoms after ACS. 


\section{METHODS}

\section{Study Sample}

Data for this study come from the Coronary Psychosocial Evaluation Studies (COPES), a series of studies that examined the association between depression and post-ACS prognosis. Details of this study have been previously described (Davidson et al., 2010). The parent study included 457 post-ACS patients who were admitted to the coronary care and cardiac care telemetry units of 3 university hospitals (Mount Sinai Medical Center, New York, NY, and Yale-New Haven Hospital and Hospital of St Raphael, New Haven, CT) between May 2003 and June 2005. The protocol complies with the Declaration of Helsinki. The Institutional Review Boards of each hospital approved the study, and informed consent was obtained from all participants after the study had been fully explained.

\section{Inclusion Criteria}

Patients were eligible if they met standard criteria for ACS (Cannon et al., 2001) (either acute MI with or without ST-segment elevation or unstable angina with documentation of coronary artery disease) verified by the study cardiologists and had eligible scores on the Beck Depression Inventory (BDI) (Beck, Ward, Mendelson, Mock, \& Erbaugh, 1961); 0-4, indicating minimal depressive symptoms, or $\geq 10$, indicating at least mild depressive symptoms) within 1 week after the index ACS hospitalization. Additionally, patients were aged 18 years or older, and spoke English or Spanish. Patients with BDI scores between 5 and 9 were not enrolled in the parent study to more clearly delineate depressed and nondepressed groups at baseline. Patients were also ineligible for study participation if they had a terminal illness (life expectancy < 1 year), had current alcohol and/or substance abuse, or had cognitive impairment as indicated by a Mini-Mental State examination score less than 24 (for educational level of 8th grade or higher) or less than 17 (for educational level of lower than 8th grade). Patients were also excluded if the screening could not be completed within 1 week of their initial hospitalization date or if they were unavailable for follow-up visits. To be eligible for this substudy, patients had to have completed the assessment of PTSD symptoms, which was initiated in November 2003, with 333 of the overall 457 patient cohort enrolled after this time point.

\section{Assessment of Posttraumatic Stress Disorder Symptoms}

Posttraumatic stress disorder symptoms were assessed 1 month after the baseline interview using the self-report Impact of Events Scale-Revised (IES-R) [23]. Participants reported on a 5-point scale ranging from 0 ("not at all") to 4 ("extremely") the extent to which they had been bothered by each of 22 PTSD symptoms (intrusions - 8 items, e.g., "Pictures about it popped into my mind;" avoidance - 8 items, e.g., "I stayed away from reminders about it;" hyperarousal - 6 items, e.g., "I was jumpy and easily startled") in the past week with reference to their index hospitalization for ACS (i.e., "Think about when you were hospitalized on (date) and we enrolled you in this study. In the past 7 days have you been bothered by any of the following in reference to that hospitalization?").

\section{Covariates}

The Global Registry of Acute Coronary Events (GRACE) risk score (Fox et al., 2006) and Charlson comorbidity index (Charlson, Szatrowski, Peterson, \& Gold, 1994)were obtained through a combination of chart review and self-report. The GRACE score is an empirically derived prognostic risk index based on clinical predictors available at the time of an ACS. GRACE variables include age, history of MI or congestive heart failure, heart rate, systolic blood pressure and serum creatinine at hospital presentation, and ST segment deviation, elevated cardiac enzymes, and percutaneous intervention (PCI) during hospitalization (Eagle 
et al., 2004). The Charlson comorbidity index consists of 22 medical conditions weighted by their associated mortality risk. The left ventricular ejection fraction (LVEF) that was most proximate to the baseline cardiac event was abstracted from the chart, and was measured quantitatively by either ventriculogram ( $43 \%$ of patients), echocardiogram $(50 \%$ of patients), or nuclear study (7\% of patients). If multiple indices were available, the ventriculogram was utilized. Values for $L V E F$ were then categorized as normal $/ \mathrm{mild}$ dysfunction (LVEF $\geq 40 \%$ ) and moderate/severe dysfunction (LVEF $<40 \%$ ). Depression symptom severity was measured with the Beck Depression Inventory (BDI) [22], a 21-item measure that assesses depression symptoms experienced in the past week.

\section{Ascertainment of Medical Outcomes}

The primary end point for this study was either the first recurrence of a major adverse cardiovascular event (MACE) [hospitalization for nonfatal MI, unstable angina, or urgent/ emergency coronary revascularization procedures (percutaneous coronary intervention, coronary artery bypass grafting, or percutaneous transluminal coronary angioplasty)] or allcause mortality (ACM) within 42 months of the baseline interview. Study participants were contacted at $1,3,6,18,30$, and 42 months either by telephone or reported to the study site in person to provide follow-up data. For any patient-reported hospitalization, supporting documentation of the event was procured from hospital records. Additionally, all recruitment hospitals were proactively searched for possible MACE hospitalizations and deaths. An end point committee consisting of 2 board-certified cardiologists, blinded to PTSD and depression status, independently reviewed and classified each hospitalization; in case of disagreement, a third board-certified cardiologist adjudicated the final end point. For participants who could not be contacted or were reported deceased by a relative, the Social Security National Death Index was searched to verify vital status.

\section{Statistical Analysis}

A total PTSD score of 33 on the IES-R has been found to best discriminate participants with a PTSD diagnosis from those without (Creamer, Bell, \& Failla, 2003). Thus, scores on each of the 3 subscales were dichotomized into high and low categories, with a score of 11 or greater classified as high, and a score of less than 11 classified as low. Previous studies of PTSD and adverse outcome in MI survivors have used a similar approach (Shemesh et al., 2000; Shemesh et al., 2001; Shemesh et al., 2004). In this study, PTSD scores were severely positively skewed, and no transformation resulted in a normal distribution of scores, so analyses with continuous PTSD scores were not conducted. Student's $t$ test for continuous variables and $\chi^{2}$ test for categorical variables were used to compare those with and without high levels of intrusion symptoms on demographic, psychiatric, and medical variables.

The primary analysis concerned the relationship between PTSD intrusion symptoms and MACE/ACM over the 41-month period between the 1-month post-ACS assessment for PTSD symptoms and the final follow-up. Patients who experienced a MACE/ACM between enrollment and the 1-month PTSD assessment were removed from analyses. We followed the recommendation that covariates be selected for inclusion a priori (Freedland et al., 2005). Based on published findings of factors that might confound the association between PTSD and MACE/ACM, demographic variables (age, sex), medical covariates (LVEF, medical comorbidities (Charlson), a clinical prognostic index (GRACE), and depression were treated as covariates. Three Cox proportional hazards models (adjusted for demographics only; demographics and medical covariates; and demographics, medical covariates, and depression) were tested to estimate the adjusted hazard ratios (HR) of postACS MACE/ACM by level of PTSD intrusion symptoms. For each model, a test of the joint statistical significance of the 3 symptom clusters is presented, as well as the significance level of each symptom cluster, controlling for the others. For scale scores, missing values 
were imputed with expected values using multiple regression. All analyses were performed using statistical software (SPSS 16.0, SPSS Inc, Chicago, IL).

\section{RESULTS}

Participants

Fourteen of 261 patients (5.4\%) who completed the IES-R at the 1-month follow-up experienced a recurrent MACE between baseline and the 1-month PTSD assessment and were removed from analyses. Of the remaining 247 patients, the mean age was 60 years (range, 25-93), 110 (45\%) were women; 199 (80\%) were White; and 21 (9\%) were Hispanic. 111 patients (45\%) were diagnosed with unstable angina; 60 patients $(24 \%)$ with ST-segment elevation MI, and 76 patients (31\%) with non ST-segment elevation MI. Using $t$ and $\chi^{2}$ tests, patients who completed the IES-R $(n=261)$ were compared with those who were enrolled after this scale was added to the protocol but who did not complete it $(n=72)$. Patients who completed the IES-R had a significantly lower GRACE score (88.5 vs 99.5, $p$ $<.05)$ and were significantly less likely to have had a recurrent MACE or ACM (19\% vs $40 \%, p<.05)$.

Overall the mean length of follow-up was 23.94 months per participant. One participant was lost to follow-up prior to study end date.

\section{PTSD Symptoms}

Mean IES-R score in the sample was 11.45 (SD, 14.92). Twenty-eight patients (11\%) met the cutoff score for "likely PTSD" (i.e., total score $\geq 33$ ); 36 (15\%) for high intrusions; 32 (13\%) for high avoidance; and 21 (9\%) for high hyperarousal. Symptom clusters of PTSD were highly associated $(r>0.80$ for continuous variables; $\varphi=.63-.69$ for dichotomized variables). Demographic and medical variables at baseline according to PTSD intrusion symptom status are shown in Table 1. As expected, patients with high PTSD intrusion scores reported higher avoidance and hyperarousal symptoms, and higher depression symptom severity. Furthermore, participants with high intrusion scores had significantly higher Charlson comorbidity scores.

\section{MACE/ACM}

During the 41 months following the 1-month assessment, 21 patients (9\%) experienced a recurrent MACE (13 UA, 4 MI, 4 emergency revascularizations) and 21 (9\%) died. Given that patients' data were censored after the first MACE occurred, the combined outcome MACE/ACM comprised 21 MACE events and 15 deaths (36 adverse events total, 15\%).

\section{Unadjusted Associations of PTSD Classification and PTSD Symptoms with MACE/ACM}

Without adjustment for covariates, PTSD classification (total IES-R score $\geq 33$ ) was associated with MACE/ACM (HR $=2.42$; 95\% confidence interval [CI], $1.10-5.35$ ). Further, as hypothesized, high intrusions (score $\geq 11$ ) was a strong predictor of MACE/ $\operatorname{ACM}(\mathrm{HR}=3.00 ; 95 \% \mathrm{CI}, 1.47-6.12)$. Overall, 11 of 36 patients (31\%) classified with high intrusions had a recurrent MACE or ACM during the follow-up period compared with 25 of 211 patients $(12 \%)$ classified with low intrusions. Neither high avoidance (HR=1.67; $\mathrm{CI}, 0.74-3.87)$ nor high hyperarousal $(\mathrm{HR}=0.85$; $\mathrm{CI}, 0.26-2.85)$ were significantly associated with MACE/ACM in unadjusted analyses.

\section{Risk-Adjusted Associations of PTSD Classification and PTSD symptoms with MACE/ACM}

After adjustment for demographic and medical covariates, a trend for an independent association of PTSD classification with MACE/ACM existed (HR=2.04; CI, .86 - 4.86), 
however, after adjustment for the effect of depression, the relationship was attenuated $(\mathrm{HR}=1.36, \mathrm{CI}=.54-3.46)$.

Table 2 presents the Cox proportional hazards regression analyses for the independent associations between PTSD symptom clusters and MACE/ACM. In the fully-adjusted model with age, sex, GRACE risk score, Charlson comorbidity index, LVEF, and depression classification, the simultaneous entry of PTSD intrusions, avoidance, and hyperarousal classification improved the model $\left(\Delta \chi^{2}=7.67 ; P=.05\right.$ ), but only intrusions (not avoidance or hyperarousal) significantly predicted recurrent MACE/ACM. Of note, in the fullyadjusted model, both depression classification and intrusions were significant predictors of MACE/ACM, beyond all other covariates (Table 2). In this model, patients classified as high on intrusions had more than 3 times greater risk of MACE/ACM $(\mathrm{HR}=3.35 ; \mathrm{CI}, 1.27$ $8.80 ; P=.02$ ) than those classified as low on intrusions (Figure 1).

We tested alternative models to ensure that these results were not dependent on our data analysis decisions. Because the hyperarousal subscale of the IES-R is comprised of only 6 items, and the intrusions and avoidance subscales are comprised of 8 items, we repeated the analyses with the hyperarousal subscale dichotomized at 7 . We chose a cutpoint of 7 as this resulted in $15 \%$ of the sample receiving a categorization as "high hyperarousal" which paralleled the prevalence of "high intrusions" and "high avoidance" using the cutpoint of 11 on the other subscales. In the fully-adjusted model with the alternate hyperarousal variable, the results were unchanged. Additionally, due to the strong positive relationship between the intrusion and hyperarousal subscale scores and the trend toward a protective effect of hyperarousal in the fully-adjusted model, we considered that including both in the model might have artificially exaggerated the effect of each. To evaluate this possibility, we tested a model without the hyperarousal subscale. The effect of intrusions remained significant and was not substantially decreased $(\mathrm{HR}=2.94 ; \mathrm{CI}, 1.06-8.24)$.

\section{DISCUSSION}

We found that a considerable proportion of patients with acute coronary syndromes develop elevated PTSD symptoms, and elevated intrusion symptoms in particular. Further, our results confirm and extend prior findings of an association between post-ACS PTSD symptoms and increased risk of recurrent MACE/ACM. In contrast to previous research (Shemesh et al., 2004) that has demonstrated a relationship between elevated levels of ACSinduced PTSD symptoms and cardiac readmission, we were able to adjust for clinical covariates including both the GRACE risk score and Charlson co-morbidity index in the current study. To our knowledge, this is the first study to highlight an independent association of ACS-induced PTSD symptoms with risk of recurrent MACE/ACM, and the first to show that the intrusion symptom cluster may specifically confer elevated risk. While previous research has shown both an unadjusted (using the IES-R) and independent association of PTSD classification (using a different PTSD measure) with cardiac readmission, we found that after adjustment for medical covariates and depression, that the hazard ratio associated with PTSD classification remained elevated but was attenuated in this still relatively small sample. Of note, however, intrusion symptoms continued to be associated with MACE/ACM even after adjustment for depression, thus demonstrating the important and unique contribution of PTSD intrusions to post-ACS event-free survival.

Consistent with our hypothesis, high intrusion symptoms consequent to ACS were associated with a greater than 3-fold increase in risk for recurrent MACE or ACM after up to 41 months of follow-up, In contrast, avoidance and hyperarousal symptoms were unrelated to post-ACS prognosis. Although some previous research (Shemesh et al., 2004) has suggested that avoidance behaviors may lead to worse medication adherence and thus 
increased risk of hospital readmission, others have found that increased avoidance symptoms are associated with lower ambulatory blood pressure (Newton et al., 2005). The non-significant protective trend for hyperarousal symptoms may appear counterintuitive, as one might expect that hyperarousal symptoms would be associated with increased autonomic activity, and thus predictive of recurrent MACE/ACM. However, no research has found a relationship between hyperarousal and MACE/ACM, and it is unclear the extent to which cardiac medications such as beta-blockers influence the experience of PTSD-related hyperarousal or the relationship between perceived hyperarousal and MACE/ACM risk.

Intrusion symptoms have consistently been related to cardiovascular outcomes and physiologic markers of increased risk, and this study suggests that they may be the most important symptoms of PTSD for the prediction of MACE or ACM. There is a sound biological basis for the relationship, in that previous research has found that intrusions are related to elevated systolic blood pressure, blood markers of endothelial dysfunction (von Kanel et al., 2008), and higher circulating levels of both catecholamines (Davidson \& Baum, 1986; Hawk, Dougall, Ursano, \& Baum, 2000) and C-reactive protein (Miller, Sutherland, Hutchison, \& Alexander, 2001). Furthermore, intrusions have been associated with elevated physiological stress response years after exposure to a traumatic event (Baum, Cohen, \& Hall, 1993), and a recent study of ambulatory cardiovascular functioning found that intrusion symptoms, but not avoidance or hyperarousal symptoms, were related to elevated mean awake ambulatory blood pressure (Newton et al., 2005).

\section{Limitations}

The present study relied on a self-report measure of PTSD symptom severity rather than on a diagnostic interview. The specific measure employed does not cover all possible PTSD symptoms, so the importance of a PTSD diagnosis for post-ACS event-free survival could not be determined. Also, patients with BDI depression scores of 5-9 were excluded from the parent study. The exclusion of these patients may have limited the variability in PTSD scores in this sample, and may have resulted in an overestimate of the true prevalence of PTSD symptoms. However, the possible range of BDI scores is 0-63, so scores between 5 and 9 are a relatively small portion of possible scores. Patients who were eligible but did not complete the IES-R had worse prognosis. Accordingly, we may have underestimated the true prevalence of elevated PTSD symptoms after ACS and the true magnitude of the PTSD to MACE/ACM relationship. Further, this study would have been stronger if we had been able to ascertain cause of death for the ACM outcome, given that PTSD due to non-ACS events has been associated with cardiac mortality.

The large ratio of predictors to MACE or ACM events in the fully-adjusted models was not ideal. Although it would have been preferable to test our hypotheses in a sample that included 10 events for every predictor, the finding that intrusions were significantly associated with MACE/ACM in the fully-adjusted model suggests that limited power was not an issue. Moreover, the consistency between the significant unadjusted association between PTSD symptoms and recurrent MACE/ACM (using a regression model in which the predictor-to-event ratio was not a concern) and the fully-adjusted association further supports our decision to control for theoretically important covariates even though the predictor-to-event ratio was not ideal. Indeed, concerns about model overfitting forced us not to consider other demographic and behavioral variables that could be thought to influence the association between intrusions and MACE/ACM (e.g., income level, smoking), but the fact that participants with high intrusions did not differ from those with low intrusions on any measured variable not accounted for in the model diminishes our concern about leaving them out. Finally, the use of dichotomous PTSD symptom variables may represent a limitation of our study. However, this categorical approach is consistent with the current classification scheme in the Diagnostic and Statistical Manual of Mental 
Disorders (Fourth Edition), and previous post-ACS PTSD research predicting adverse medical outcomes has likewise used dichotomized scoring (Shemesh et al., 2006; Shemesh et al., 2001; Shemesh et al., 2004).

Notwithstanding the aforementioned possible limitations, this study is by far the largest to demonstrate an association between PTSD symptoms after ACS and recurrent MACE/ACM. It is also the first to include mortality among the primary endpoints.

\section{Conclusion}

For many patients, an ACS is sufficiently traumatic to elicit PTSD symptoms. The findings of the present study demonstrate that patients who have intrusive, emotionally-charged thoughts, nightmares, or flashbacks related to their ACS may be at especially high risk for MACE recurrence and mortality. Further study is needed to better characterize these patients at high risk, to better illuminate the mechanisms underlying the relationship between PTSD and CVD, and to assess the benefit of possible interventions.

\section{Acknowledgments}

Data collection and manuscript preparation were financially supported by grants HC-25197 and HL088117, from the National Heart, Lung and Blood Institute, Bethesda, Maryland. Dr. Edmondson is supported by grant KM1CA156709. Dr Kronish is supported by grant 1K23HL098359 from the National Heart, Lung, and Blood Institute.

\section{Abbreviations}

$\begin{array}{ll}\text { ACM } & \text { all-cause mortality } \\ \text { ACS } & \text { acute coronary syndrome } \\ \text { CVD } & \text { cardiovascular disease } \\ \text { GRACE } & \text { Global Registry of Acute Coronary Events } \\ \text { IES-R } & \text { Impact of Events Scale-Revised } \\ \text { LVEF } & \text { left ventricular ejection fraction } \\ \text { MACE } & \text { major adverse cardiac event } \\ \text { MI } & \text { myocardial infarction } \\ \text { PTSD } & \text { posttraumatic stress disorder } \\ \text { UA } & \text { unstable angina }\end{array}$

\section{References}

Ayers S, Copland C, Dunmore E. A preliminary study of negative appraisals and dysfunctional coping associated with post-traumatic stress disorder symptoms following myocardial infarction. British Journal of Health Psychology. 2009; 14:459-71. [PubMed: 18789186]

Baum A, Cohen L, Hall M. Control and intrusive memories as possible determinants of chronic stress. Psychosomatic Medicine. 1993; 55:274-86. [PubMed: 8346335]

Beck AT, Ward CH, Mendelson M, Mock J, Erbaugh J. An inventory for measuring depression. Archives of General Psychiatry. 1961; 4:561-71. [PubMed: 13688369]

Cannon CP, Battler A, Brindis RG, Cox JL, Ellis SG, Every NR, Flaherty JT, Harrington RA, Krumholz HM, Simoons ML, Van De Werf FJ, Weintraub WS, Mitchell KR, Morrisson SL, Anderson HV, Cannom DS, Chitwood WR, Cigarroa JE, Collins-Nakai RL, Gibbons RJ, Grover FL, Heidenreich PA, Khandheria BK, Knoebel SB, Krumholz HL, Malenka DJ, Mark DB, McKay CR, Passamani ER, Radford MJ, Riner RN, Schwartz JB, Shaw RE, Shemin RJ, Van Fossen DB, 
Verrier ED, Watkins MW, Phoubandith DR, Furnelli T. American College of Cardiology key data elements and definitions for measuring the clinical management and outcomes of patients with acute coronary syndromes. A report of the American College of Cardiology Task Force on Clinical Data Standards (Acute Coronary Syndromes Writing Committee). Journal of the American College of Cardiology. 2001; 38:2114-30. [PubMed: 11738323]

Charlson M, Szatrowski TP, Peterson J, Gold J. Validation of a combined comorbidity index. Journal of Clinical Epidemiology. 1994; 47:1245-51. [PubMed: 7722560]

Creamer M, Bell R, Failla S. Psychometric properties of the Impact of Event Scale - Revised. Behaviour Research \& Therapy. 2003; 41:1489-96. [PubMed: 14705607]

Davidson KW, Burg MM, Kronish IM, Shimbo D, Dettenborn L, Mehran R, Vorchheimer D, Clemow L, Schwartz JE, Lesperance F, Rieckmann N. Association of Anhedonia With Recurrent Major Adverse Cardiac Events and Mortality 1 Year After Acute Coronary Syndrome. Archives of General Psychiatry. 2010; 67:480-488. [PubMed: 20439829]

Davidson LM, Baum A. Chronic stress and posttraumatic stress disorders. Journal of Consulting \& Clinical Psychology. 1986; 54:303-8. [PubMed: 3722557]

Doerfler, LA.; Paraskos, JA. Posttraumatic Stress Disorder (PTSD) Following Myocardial Infarction or Cardiac Surgery. In: Allen, R.; Fisher, J., editors. Heart and Mind: The Practice of Cardiac Psychology. Washington, DC: American Psychological Association; 2011.

Doerfler LA, Paraskos JA, Piniarski L. Relationship of quality of life and perceived control with posttraumatic stress disorder symptoms 3 to 6 months after myocardial infarction. Journal of Cardiopulmonary Rehabilitation. 2005; 25:166-72. [PubMed: 15931021]

Doerfler LA, Pbert L, DeCosimo D. Symptoms of posttraumatic stress disorder following myocardial infarction and coronary artery bypass surgery. General Hospital Psychiatry. 1994; 16:193-9. [PubMed: 8063086]

Eagle KA, Lim MJ, Dabbous OH, Pieper KS, Goldberg RJ, Van de Werf F, Goodman SG, Granger CB, Steg PG, Gore JM, Budaj A, Avezum A, Flather MD, Fox KA. A validated prediction model for all forms of acute coronary syndrome: estimating the risk of 6-month postdischarge death in an international registry. Journal of the American Medical Association. 2004; 291:2727-33. [PubMed: 15187054]

Falger PR, Op den Velde W, Hovens JE, Schouten EG, De Groen JH, Van Duijn H. Current posttraumatic stress disorder and cardiovascular disease risk factors in Dutch Resistance veterans from World War II. Psychotherapy \& Psychosomatics. 1992; 57:164-71. [PubMed: 1410192]

Fox KAA, Dabbous OH, Goldberg RJ, Pieper KS, Eagle KA, Van de Werf F, Avezum Á, Goodman SG, Flather MD, Anderson FA, Granger CB. Prediction of risk of death and myocardial infarction in the six months after presentation with acute coronary syndrome: prospective multinational observational study (GRACE). British Medical Journal. 2006; 333:1091. [PubMed: 17032691]

Freedland KE, Babyak MA, McMahon RJ, Jennings JR, Golden RN, Sheps DS. Statistical Guidelines for Psychosomatic Medicine. Psychosom Med. 2005; 67:167.

Gamper G, Willeit M, Sterz F, Herkner H, Zoufaly A, Hornik K, Havel C, Laggner AN. Life after death: posttraumatic stress disorder in survivors of cardiac arrest--prevalence, associated factors, and the influence of sedation and analgesia. Critical Care Medicine. 2004; 32:378-83. [PubMed: 14758151]

Gander ML, von Kanel R. Myocardial infarction and post-traumatic stress disorder: frequency, outcome, and atherosclerotic mechanisms. European Journal of Cardiovascular Prevention \& Rehabilitation. 2006; 13:165-72. [PubMed: 16575268]

Hawk LW, Dougall AL, Ursano RJ, Baum A. Urinary catecholamines and cortisol in recent-onset posttraumatic stress disorder after motor vehicle accidents. Psychosomatic Medicine. 2000; 62:423-34. [PubMed: 10845356]

King DW, Leskin GA, King LA, Weathers FW. Confirmatory factor analysis of the clinicianadministered PTSD Scale: Evidence for the dimensionality of posttraumatic stress disorder. Psychological Assessment. 1998; 10:90-96.

Kubzansky LD, Koenen KC. Is posttraumatic stress disorder related to development of heart disease? An update. Cleveland Clinic Journal of Medicine. 2009; 76 (Suppl 2):S60-5. [PubMed: 19376986] 
Ladwig KH, Schoefinius A, Dammann G, Danner R, Gurtler R, Herrmann R. Long-acting psychotraumatic properties of a cardiac arrest experience. American Journal of Psychiatry. 1999; 156:912-9. [PubMed: 10360132]

Miller RJ, Sutherland AG, Hutchison JD, Alexander DA. C-reactive protein and interleukin 6 receptor in post-traumatic stress disorder: a pilot study. Cytokine. 2001; 13:253-5. [PubMed: 11237435]

Newton TL, Parker BC, Ho IK. Ambulatory cardiovascular functioning in healthy postmenopausal women with victimization histories. Biological Psychology. 2005; 70:121-130. [PubMed: 16168256]

Shalev AY, Freedman S, Peri T, Brandes D, Sahar T, Orr SP, Pitman RK. Prospective study of posttraumatic stress disorder and depression following trauma. American Journal of Psychiatry. 1998; 155:630-7. [PubMed: 9585714]

Shemesh E, Koren-Michowitz M, Yehuda R, Milo-Cotter O, Murdock E, Vered Z, Shneider BL, Gorman JM, Cotter G. Symptoms of Posttraumatic Stress Disorder in Patients Who Have Had a Myocardial Infarction. Psychosomatics. 2006; 47:231-239. [PubMed: 16684940]

Shemesh E, Lurie S, Stuber ML, Emre S, Patel Y, Vohra P, Aromando M, Shneider BL. A pilot study of posttraumatic stress and nonadherence in pediatric liver transplant recipients. Pediatrics. 2000; 105:E29. [PubMed: 10654989]

Shemesh E, Rudnick A, Kaluski E, Milovanov O, Salah A, Alon D, Dinur I, Blatt A, Metzkor M, Golik A, Verd Z, Cotter G. A prospective study of posttraumatic stress symptoms and nonadherence in survivors of a myocardial infarction (MI). General Hospital Psychiatry. 2001; 23:215-22. [PubMed: 11543848]

Shemesh E, Yehuda R, Milo O, Dinur I, Rudnick A, Vered Z, Cotter G. Posttraumatic stress, nonadherence, and adverse outcome in survivors of a myocardial infarction. Psychosomatic Medicine. 2004; 66:521-6. [PubMed: 15272097]

von Kanel R, Hari R, Schmid JP, Wiedemar L, Guler E, Barth J, Saner H, Schnyder U, Begre S. Nonfatal cardiovascular outcome in patients with posttraumatic stress symptoms caused by myocardial infarction. Journal of Cardiology. 2011

von Kanel R, Hepp U, Traber R, Kraemer B, Mica L, Keel M, Mausbach BT, Schnyder U. Measures of endothelial dysfunction in plasma of patients with posttraumatic stress disorder. Psychiatry Research. 2008; 158:363-73. [PubMed: 18252265] 


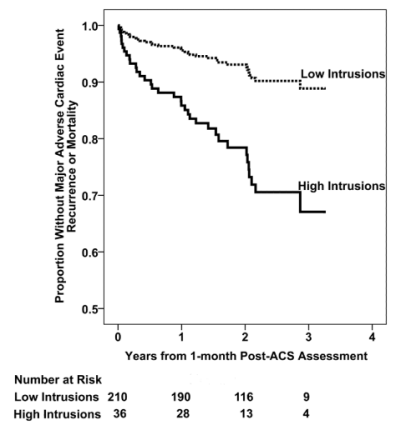

Figure 1.

Cox proportional hazards regression analysis predicted curve for patients with and without high levels of intrusion symptoms, adjusted for age, sex, Charlson comorbidity index score, Global Registry of Acute Coronary Events risk score, left ventricular ejection fraction, and depression symptom severity according to the Beck Depression Inventory. Also, the number of patients in each group who were at risk for MACE/ACM at each year are presented below the figure. Abbreviation: ACS, acute coronary syndrome. 
Table 1

Demographics, clinical characteristics, and outcomes of 247 patients with acute coronary syndromes by PTSD intrusions group ${ }^{a}$

\begin{tabular}{|c|c|c|}
\hline \multirow[b]{2}{*}{ Characteristic } & \multicolumn{2}{|c|}{ PTSD intrusions } \\
\hline & High $(n=36)$ & Low $(n=211)$ \\
\hline Age, y & $57.1 \pm 12.2$ & $60.7 \pm 12.9$ \\
\hline Male, $n(\%)$ & $24(67)$ & $112(53)$ \\
\hline White, $n(\%)$ & $28(78)$ & $171(81)$ \\
\hline Hispanic, $n(\%)^{b}$ & $7(19)$ & $15(7)$ \\
\hline Mean income & $\$ 35-50,000$ & $\$ 35-50,000$ \\
\hline Body mass index & $28.55(8.16)$ & 28.99 \\
\hline GRACE risk score & $80.2 \pm 30.6$ & $89.5 \pm 32.5$ \\
\hline ST-segment elevation MI, $n(\%)$ & $8(23)$ & $51(24)$ \\
\hline Non-ST-segment elevation MI, $n(\%)$ & $9(26)$ & $70(33)$ \\
\hline Unstable angina, $n(\%)$ & $18(50)$ & $91(43)$ \\
\hline Charlson comorbidity index ${ }^{b}$ & $2.1 \pm 1.9$ & $1.3 \pm 1.6$ \\
\hline Percutaneous transluminal coronary angioplasty, $n(\%)$ & $30(84)$ & $158(75)$ \\
\hline C-reactive protein at baseline $(n=145)$ & $24.9 \pm 33.0$ & $26.1 \pm 41.5$ \\
\hline C-reactive protein at 1-month $(n=120)$ & $7.3 \pm 8.6$ & $5.7 \pm 9.6$ \\
\hline Left ventricular ejection fraction & $53.8 \pm 12.5$ & $52.9 \pm 14.1$ \\
\hline Peak troponin & $73.9 \pm 148.2$ & $116.3 \pm 303.6$ \\
\hline Peak creatinine kinase & $1011.2 \pm 1821.8$ & $1019.5 \pm 1545.6$ \\
\hline Left ventricular ejection fraction $\geq 40, n(\%)$ & $5(13)$ & $34(16)$ \\
\hline Diabetes, $n(\%)$ & $15(42)$ & $62(30)$ \\
\hline Hypertension, $n(\%)$ & $32(89)$ & $174(85)$ \\
\hline Endorsed recent alcohol consumption, $n(\%)$ & $9(27)$ & $59(30)$ \\
\hline Number of drinks in pre-event week & $3.44(4.25)$ & $3.47(3.52)$ \\
\hline Smoking history, $n(\%)$ & $26(72)$ & $121(57)$ \\
\hline Antidepressant use, $n(\%)^{c}$ & $12(34)$ & $29(14)$ \\
\hline BDI score ${ }^{c}$ & $16.4 \pm 12.1$ & $7.3 \pm 7.6$ \\
\hline BDI score $\geq 10, n(\%)^{c}$ & $28(78)$ & $87(41)$ \\
\hline IES-R score ${ }^{c}$ & $21.1 \pm 12.7$ & $6.3 \pm 7.5$ \\
\hline Total score $\geq 33, n(\%)^{c}$ & $26(72)$ & $2(1)$ \\
\hline Intrusions $c$ & $16.3 \pm 4.3$ & $2.2 \pm 2.7$ \\
\hline Avoidance $^{c}$ & $14.5 \pm 6.1$ & $2.6 \pm 3.5$ \\
\hline Avoidance $\geq 11, n(\%)^{c}$ & $25(69)$ & $6(3)$ \\
\hline Hyperarousal $^{c}$ & $10.4 \pm 5.3$ & $1.5 \pm 2.4$ \\
\hline Hyperarousal $\geq 11, n(\%)^{c}$ & $18(50)$ & $2(1)$ \\
\hline
\end{tabular}

Abbreviations: BDI, Beck Depression Inventory; IES-R, Impact of Events Scale-Revised; PTSD, posttraumatic stress disorder; MI, myocardial infarction; GRACE, Global Registry of Acute Coronary Events 
${ }^{a}$ Values are mean \pm SD unless indicated otherwise.

${ }^{b}{ }_{P}<.05$.

${ }^{c} P<.01$. 


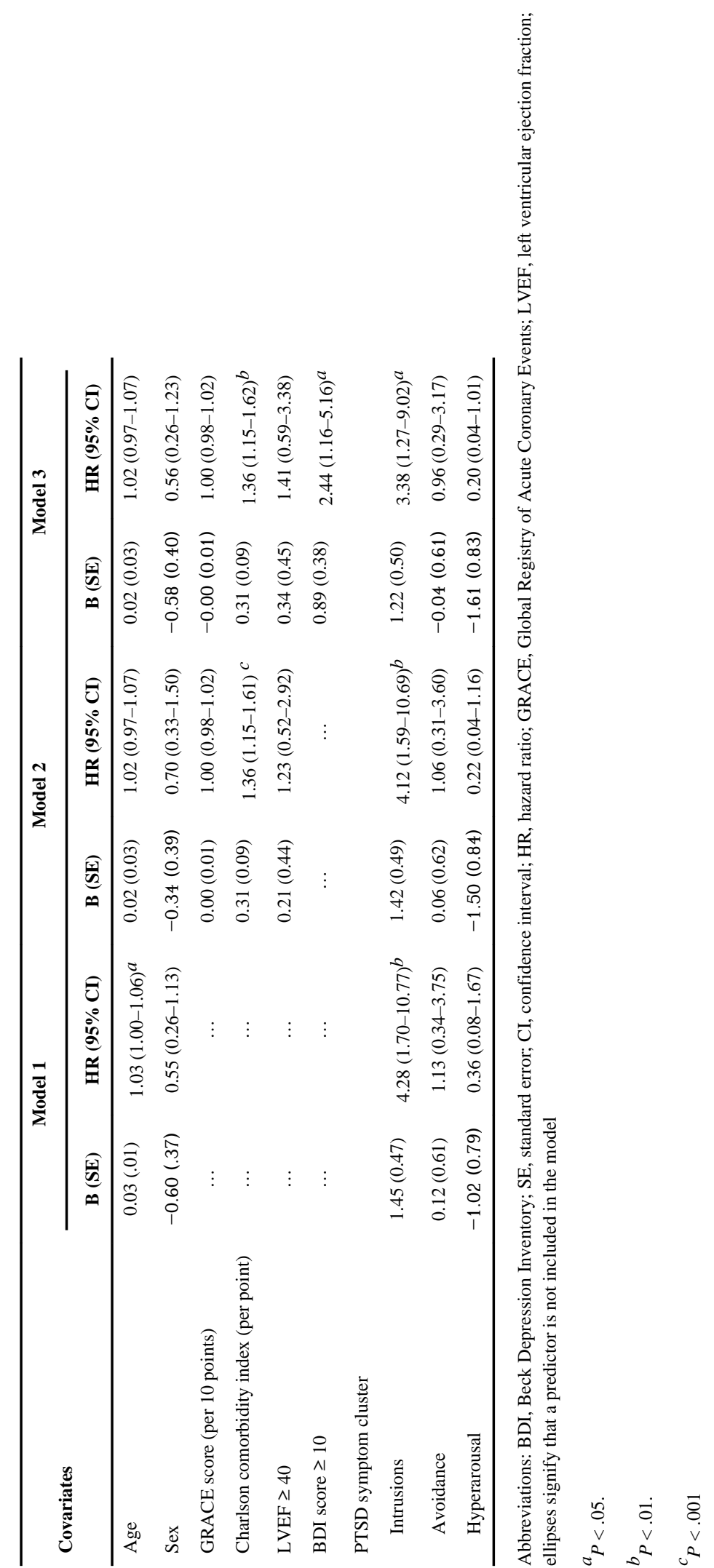

